\title{
Optimal Spacing of "Covered" and "Exposed" Time Intervals in a Stochastic Process with High Penalty Costs: Applications to Parking and Insurance
}

\author{
Behrouz Bakhtiari \\ Operations Management Area, DeGroote School of Business, McMaster University, Hamilton, Ontario, Canada L8S 4M4, \\ email:bakhtib@mcmaster.ca \\ Emre Berk \\ Department of Management, Faculty of Business Administration, Bilkent University, Bilkent University, 06800 Bilkent, Ankara, \\ Turkey, Bilkent, email: eberk@bilkent.edu.tr \\ Elkafi Hassini \\ Operations Management Area, DeGroote School of Business, McMaster University, Hamilton, Ontario, Canada L8S 4M4, \\ email: hassini@mcmaster.ca \\ Mahmut Parlar \\ Operations Management Area, DeGroote School of Business, McMaster University, Hamilton, Ontario, Canada L8S 4M4, \\ email:parlar@mcmaster.ca
}

\begin{abstract}
This paper studies a class of policies for a stochastic process that is constituted of several time intervals of total time $T$. The intervals can be covered (or insured) at a pay-per-use rate or exposed (uninsured) with the risk of a large penalty. A decision maker has the three options: (i) Pay the user fee for the full period, (ii) not pay at all, and (iii) sporadically pay a user fee leaving an uncovered period at the end of each covered one. The penalty risk is assumed to occur during an uncovered interval according to a Poisson process. We present the expected cost model and find the optimal coverage policy. We present conditions under which it is always optimal to pay in full or not pay at all to minimize the expected total cost. Finally, we relax two assumptions and allow for the consideration of setup costs for every time the decision maker pays the coverage fees as well as a random duration $T$ and derive new conditions for optimal strategies. Possible application of our model is paying parking meter fees and deciding between self-insuring one's property and buying full (or partial) insurance coverage.
\end{abstract}

Keywords: Poisson processes; parking policies; insurance

This manuscript was handled and accepted by Bernard Gendron, former Editor-in-Chief.

\section{INTRODUCTION}

Most people who park their cars in a parking space to attend a show, or to take their children to music lessons face a decision problem: How much money to pay for the parking space? Some may choose to pay for the full period, others may choose to pay partially or not at all and risk a parking ticket. A similar decision problem arises when deciding between self-insuring one's property and buying full (or partial) insurance coverage. If the property is self-insured (corresponding to not paying the parking fee), then the property owner assumes full responsibility for the costs arising from a randomly occurring event such as fire. If the property is fully insured for a specific length of time (corresponding to paying the full parking fee), then any costly random events are covered by the insurance company.

Motivated by the above practical examples, in this paper we propose models for managing risk and minimizing cost to the individual in such settings. Using the parking example, the main idea of the problem is as follows: A patron needs to park her car at a parking space for a known duration of $T$ time units, say, hours. We will later relax this assumption and extend the model to the case where $T$ is random. The parking space has a parking rate of $c_{I}>0$ dollars per unit of time. We note that we do not consider variations in parking rates by time of day or day of the week. In addition, we assume that there are no restrictions on 
maximum parking durations. The patron is willing to visit the parking space for a maximum of $N$ times to feed the meter for $u$ units of time and leave a duration of $v$ unpaid, each time. Thus, $T$ is divided into $N$ intervals of equal duration $L$, where $u$ is the covered interval and $v$ is the exposed interval within $L(=u+v)$ so that $T=N L$. If the parking inspector visits the spot during $v$, the patron will be ticketed and otherwise, she would be safe until the next visit of the inspector or until time $T$ is reached, whichever comes first. If the patron is ticketed, the problem ends and a ticket cost of $c_{P}>0$ has to be paid by the patron. Finally, the patron returns to her car at time $T$ and drives away. We note that in practice a parking fee would only allow a discrete set of durations. For simplicity we assume that $u$ can take any non-negative value.

Larson (1970) points out that police services have recognized the potential of random patrols. We assume that the parking inspector visits the spot according to a Poisson process with parameter $\lambda>0$. We are interested in finding the optimal value of the duration of coverage at each visit, $u$, and the number of visits to the car, $N$, that would minimize the expected total cost to the patron. It is worth pointing out that we are studying a simple class of policies where the periods $u$ and $v$ are fixed. A more general problem where these intervals may be time-dependent is under investigation by the authors.

In this study we consider the above problem of optimal parking fee payment and formulate it to find the optimal solution. The objective of the car owner is to minimize the expected cost of "insurance" plus the expected cost of "penalty" during the time of stay at the parking space. We divide the total time of stay into equal subintervals and find the optimal duration of time in each subinterval in order to minimize the expected total cost. We show that for one or two subintervals, the optimal policy calls for either insuring fully, or not insuring at all. For more subintervals, we provide sufficient conditions under which either paying in full, or paying nothing is optimal. The remainder of this paper is organized as follows: We review the related literature in Section 2. In Section 3 we present the assumptions and develop the expected cost functions for the insurance coverage and the penalty cost. The optimization model and its analysis are provided in Section 4 and numerical examples are given in Section 5. In Section 6 two extensions are provided to make the model more general. The paper ends with a brief summary and conclusions in Section 7.

\section{RELATED LITERATURE}

In this paper, we are taking the perspective of a user of the parking service. However, some of the insights are also useful to policy makers. For example, from the perspective of a municipal parking services, they may be interested in finding the optimal pattern for parking inspection visits as well as parking rates to increase revenue and/or enforce parking legislations. In Section 5 we perform sensitivity analysis on the problem parameters that can be used by authorities to study the impact of parking fees, fines and staff patrolling frequency on the driver behaviour.
Rojas (2006) modelled drivers' parking choice and found that it is elastic with respect to price, but not distance. Using this model they developed a revenue management model to dynamically change parking prices so as to maximize revenue for the parking lot operator. Cassady and Kobza (1998) have studied the decision of selecting a parking space by considering the driver's walking and driving efforts. The authors focus on free parking lots such as those of shopping centres. Thus, parking cost was not a factor for the parking space selection problem. Some authors have also studied the effect of raising parking fees on drivers' parking behaviours. For further reviews in this context see, Verhoef et al. (1995), Miller and Everett (1982), Willson and Shoup (1990), and Shoup (1995). Other studies dealt with parking rates and how they relate to controlling traffic congestion, land use and emissions; see, for example, Arnott and Inci (2006), Arnott and Rowse (1999), Arnott and Rowse (2009), D'Acierno et al. (2006), Hess (2001), Shoup (1997), Shoup (1999), and Willson (1995). Elliot and Wright (1982), Fishman and Miguel (2007), and Petiot (2004) have provided empirical evidence on the effectiveness of parking enforcement.

The model discussed in this paper is concerned with a simple decision problem of an individual with somewhat minor consequences. However, as mentioned above, it can be applied in other contexts such as where decisions should be made about buying or not buying insurance coverage. In other words, an individual can compare the expected total costs associated with buying insurance and covering herself over a period of time and not buying insurance and taking the risk of loss with a certain probability. There are some studies in the literature that address this issue and find conditions for buying and not buying insurance. For example, Hung (2009) takes the case of flood insurance contracts and shows that uncertainty is an important factor in the process of decision making for buying or not buying high-cost, low-probability insurance. He finds that attributes like estimated risk of flood and estimated loss value in case of a flood are important factors in determining the purchase of flood insurance coverages. Kunreuther (1996) explains why some individuals are reluctant to purchase insurance coverage, or alternatively lose their interest in maintaining insurance after a certain period of time. He argues that individuals may abstain from purchasing insurance when, a) they underestimate the risk of disasters, or b) their anticipated duration of stay in their current location is lower than a certain threshold that would justify buying coverage, or c) the premium that they need to pay per time unit does not justify purchasing insurance based on the probability of disaster and the duration of stay. Our model builds on the findings of Hung (2009) and Kunreuther (1996) by allowing the decision maker to model uncertainty (in risk or police arrival) and provide decisions rules to support some of the empirical observations from the insurance literature.

Other authors have studied the relationship between the purchase of insurance and the price of coverage as well as the probability of loss. For instance, Ganderton et al. (2000) have shown 
through a set of experiments how the probability of purchasing insurance increases as the perceived probability of loss increases. It will also increase if the cost of loss increases or if the cost of insurance coverage falls. For similar studies, see Dragos (2014), Hung (2009), Lewis and Nickerson (1989), Meier (1999), Mossin (1968), Sherden (1984) and Slovic et al. (1977).

\section{MODEL DEVELOPMENT}

As indicated in the Introduction, we are considering a problem where the car owner will park her car for a total of $T$ time units, say, hours. Let us assume that she is willing to return to her car for a total of $N$ times to to pay for parking where $1 \leq N \leq \hat{N}$ and $\hat{N}$ is an upper bound on $N$. If so, she must decide the amount of coverage during each subinterval of length $L=T / N$. She can either pay the full amount at the beginning in which case there will not be any need to return to the parking space; in that case, by default, $N=1$. Alternatively, she can pay a smaller amount to cover herself for $u$ time units during each subinterval $L$, and take a risk and not pay for $v$ time units within $L(=u+$ $v$ ). The parking fee (insurance rate) is $c_{I}$ per time unit.

A parking inspector visits the parking space according to a Poisson process with rate $\lambda$. Since the car is "exposed" during intervals of length $v$ in each cycle, we have,

$$
\operatorname{Pr}(\text { Not getting caught in } v)=p_{0}(v)=e^{-\lambda v},
$$

and

$$
\operatorname{Pr}(\text { Getting caught in } v)=1-p_{0}(v)=q(v)=1-e^{-\lambda v} .
$$

There is a penalty of $c_{P}$ if the car is not "covered" during $v$ and the inspector spots it and tickets the car. If that happens the process stops. The question is; what are the optimal values of $u$, $v$ and $N$ that minimize the expected total cost?

\subsection{Expected cost of Parking fee (Insurance Coverage) $I(q, N)$}

We define the random variable $K$ as the number of times the car owner will pay for parking fees (including the initial feeding) with the probability mass function (p.m.f.) $f(k)$. Since the car owner is willing to pay parking fees a maximum of $N$ times, the random variable $K$ assumes the values $1, \ldots, N$.

To illustrate the determination of the p.m.f. consider a special case where $N=4$. The outcome $K=1$ occurs when the parking inspector arrives during $v$ of the first interval and finds that the coverage has expired and writes a ticket which stops the process. This happens with probability $f(1)=q$, where $q \equiv q(v)=1$ $-p_{0}(v)$. Similarly, we have $K=2$, if the car owner is not caught in the first interval (with probability $1-q$ ) but is caught in the second (with probability $q$ ) which happens with probability $f(2)$ $=(1-q) q$. Likewise, we have $K=3$, if the parking inspector does not arrive during $v$ of the first two intervals, but arrives during $v$ of the third interval and tickets the car which has the probability $f(3)=(1-q)^{2} q$. The case of $K=4$ occurs if the car

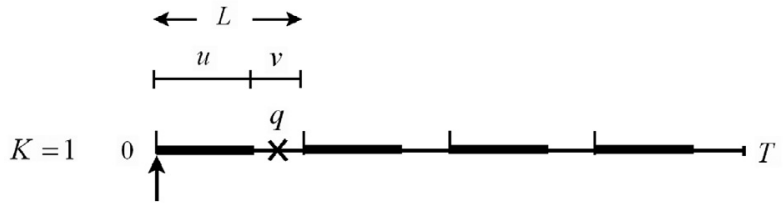

$K=2$

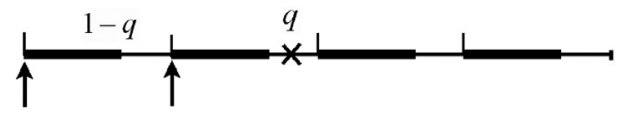

$K=3$

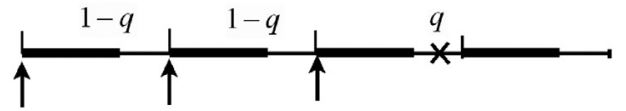

$K=4$

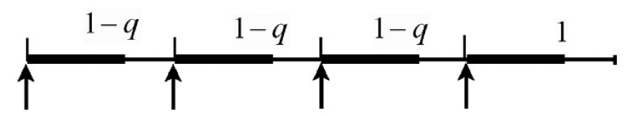

Figure 1. The random variable $K$ is defined as the number of times the car owner will pay for parking, including the initial payment. In this example, $K=1, \ldots, 4$ since $N=4$. In this figure the arrows represent the time points at which the parking fee is paid, and the cross $\times$ corresponds to the arrival of the parking inspector during time interval $v$ (when the car owner receives a ticket).

owner is not caught in any of the first three intervals with probability $f(4)=(1-q)^{3} \cdot 1$ (regardless of what happens in the last interval). See Figure 1 for a graphical explanation of this case with $N=4$. Thus, $K$ is equivalent to $\min \left(K_{\infty}, N\right)$ where $\mathrm{K}_{\infty}$ has a geometric distribution with parameter $q$.

Thus, in general, for any integer $N$ we have,

$$
\begin{aligned}
& f(k)=(1-q)^{k-1} q, \quad k=1, \cdots, N-1 \\
& f(N)=(1-q)^{N-1} .
\end{aligned}
$$

A routine calculation shows that the expected value of $K$ is

$$
\kappa(q, N) \equiv E(K)=\sum_{k=1}^{N} k f(k)=\frac{1-(1-q)^{N}}{q} .
$$

We note here that $q=q(v)$ cannot exceed unity, but there is a natural upper bound (which is less than 1) for this quantity arising from the relation $N L=T$. Since $q=1-e^{-\lambda v}$ and $v \leq L=T / N$, it follows that $1-e^{-\lambda T / N}$ is the natural upper bound for $q$, i.e., the problem is to minimize a suitable objective function subject to $0 \leq q \leq \hat{q}$, where,

$$
\hat{q}=1-e^{-\lambda T / N}
$$

We note that in effect we have made a change of variables from $u$ and $v$ to $q$. To recuperate the values $u$ and $v$ we will make use of the equalities $q=1-e^{-\lambda v}$ and $u+v=T / N$. This change of variables reduce the dimensionality of the optimization problem and will allow us to develop a solution procedure for the problem. 
The following lemma presents some properties of the expected value function $\kappa(q, N) \equiv E(K)$.

Lemma 1 For $N=1$, the expected number of times the car owner will pay parking is a constant $\kappa(q, 1)=1$, and for $N \geq 2$, $\kappa(q, N)$ is strictly decreasing in $q$.

Proof. The statement for $N=1$ is obvious. When $N \geq 2$, we differentiate $\kappa(q, N)$ w.r.t. $q$ and obtain, after some simplifications

$$
\kappa^{\prime}(q, N)=\frac{(1-q)^{N-1}[1+(N-1) q]-1}{q^{2}} .
$$

To show that this quantity is always negative (thus $\kappa$ is decreasing), we focus on the term $\psi(q)=(1-q)^{N-1}[1+(N-1) q]$ in the numerator and show that it is always less than 1 . We have $\psi(0)=1$ and $\psi(1)=0$. Differentiating $\psi(q)$, we have

$$
\psi^{\prime}(q)=-\frac{(1-q)^{N-1}(N-1) N q}{1-q}<0 .
$$

Thus, $\psi(q)$ is monotone decreasing and always varies between 1 and 0 , implying that $\kappa^{\prime}(q, N)<0$ and so $\kappa(q, N)$ is monotone decreasing in $q$. Moreover, at the endpoints we find that

$$
\lim _{q \rightarrow 0^{+}} \kappa(q, N)=N, \quad \text { and } \quad \kappa(\hat{q}, N)=\frac{e^{\lambda T / N}\left(1-e^{-\lambda T}\right)}{e^{\lambda T / N}-1}>1,
$$

where the last result follows from the fact that $\kappa(1, N)=1$ and that $\hat{q}<1$.

Thus, the expected number of times a parking fee is paid decreases as the uncovered intervals, $v$, become longer. Lemma 1 shows that since $\lim _{q \rightarrow 0^{+}} \kappa(q, N)=N$, if $q=0$, and thus $v=0$ and $u=L$, the car owner will be paying parking fees exactly $N$ times and at each feeding she will deposit sufficient funds to cover herself completely for $L$ time units in each subinterval.

If we define $I(q, N)$ as the "insurance" cost, i.e., the expected cost of the parking fee paid by the car owner, then we have,

$$
I(q, N)=c_{I}[\kappa(q, N) u(q, N)]
$$

where $c_{I}>0$ is the cost of insurance (parking fee) per time unit, $\kappa(q, N) \equiv E(K)$ is the expected number of times the car owner will pay parking fees, and $u(q, N)$ is the length of time the car is "insured" in each cycle.

To express $u(q, N)$ in terms of its arguments, recall that $q(v)=$ $1-e^{-\lambda v}$, so that $v=-(1 / \lambda) \ln (1-q)$. Since $u+v=T / N$, we find

$$
u(q, N)=\frac{T}{N}-v=\frac{T}{N}+\frac{1}{\lambda} \ln (1-q) .
$$

Thus,

$$
I(q, N)=c_{I}\left[\frac{1-(1-q)^{N}}{q}\right]\left[\frac{T}{N}+\frac{1}{\lambda} \ln (1-q)\right]
$$

is the expected cost incurred paying for parking fees (i.e., buying insurance coverage).
Lemma 2 The length of time the car is covered by insurance, $u(q, N)$, is decreasing in $q$ for any $N \geq 1$.

Proof. Differentiating $u(q, N)$, we have

$$
u^{\prime}(q, N)=-\frac{1}{\lambda(1-q)}<0 .
$$

Thus, $u(q, N)$ is decreasing in $q$. We also find that, at the endpoints,

$$
u(0, N)=\frac{T}{N}, \quad \text { and } \quad u(\hat{q}, N)=0 .
$$

That is, if $q=0$, we also have $v=0$, so $u=T / N=L$, as should be expected. Similarly, if $q=\hat{q}$, then the car owner is not paying any parking fees, so $u$ must equal zero.

Remark 1 The result found in Lemma 2 should be expected since $q=1-e^{-\lambda v}$, i.e., $q$ is increasing in $v$, and thus it must be inversely related to $u$. That is, higher values of $q$ imply higher values of $v$, which in turn imply lower values of $u$.

Having discovered in Lemma 1 and Lemma 2 that both $\kappa(q$, $N)$ and $u(q, N)$ are decreasing in $q$, we now show that the insurance cost function also possesses the same property.

Proposition 1 The insurance cost function $I(q, N)$ is decreasing in $q$ for any $N \geq 1$.

Proof. Differentiating $I(q, N)$ w.r.t. to $q$, we have

$$
I^{\prime}(q, N)=c_{I}\left(\kappa^{\prime} u+\kappa u^{\prime}\right) .
$$

But since both $\kappa^{\prime}<0$ and $u^{\prime}<0$ (from Lemma 1 and Lemma 2, respectively), it follows that $I^{\prime}(q, N)<0$ since $\kappa(q, N)$ and $u(q, N)$ are positive functions.

Remark 2 Suppose $I(q, N)$ is the only term in the objective function, i.e., the parking inspector never checked the parking space where the car is parked. It follows from Proposition 1 that since $I(q, N)$ is decreasing, it is always optimal for the car owner to choose $q^{*}=\hat{q}$, i.e., $v^{*}=L$, and to never pay for parking.

\subsection{Expected cost of the fine (parking ticket penalty) $P$ $(q, N)$}

To calculate the expected cost of receiving a ticket from the parking inspector, we define $c_{P}$ as the cost of the ticket. The following lemma will aid in computing the expected cost of receiving a parking ticket during the time interval $T$.

Lemma 3 The probability of getting caught and receiving a parking ticket during $T$ is $1-(1-q)^{N}$.

Proof. Recall that $q=q(v)$ is the probability of getting caught in an interval of length $v$ during $L$. Since there are $N$ intervals of length $L,(1-q)^{N}$ is the probability of not getting caught in any of the $N$ intervals. Thus, $1-(1-q)^{N}$ is the probability of getting caught in at least one of the $N$ intervals, i.e., during $T$.

The expected cost of the fine is then

$$
P(q, N)=c_{P}\left[1-(1-q)^{N}\right],
$$

The following lemma establishes concavity of $P(q, N)$. 
Lemma $4 P(q, N)$ is increasing concave in $q$.

Proof. It is easy to see that when $N=1$, the penalty cost function is $P(q, N)=c_{P} q$ which is increasing and linear in $q$. For $N \geq 2$, the penalty cost function is increasing concave in $q$ since

$$
\begin{aligned}
P^{\prime}(q, N) & =c_{P}(1-q)^{N-1} N>0, \\
P^{\prime \prime}(q, N) & =-c_{P}(1-q)^{N-2} N(N-1)<0 .
\end{aligned}
$$

Lemma 4 implies that

$$
P^{\prime \prime \prime}(q, N)=c_{P}(1-q)^{N-3} N(N-1)(N-2)>0,
$$

thus $P^{\prime}(q, N)$ is a decreasing convex function when $N \geq 3$.

\section{OPTIMIZATION MODEL}

The expected total cost $C(q, N)$ is the sum of the expected insurance (parking fee) cost and the expected penalty cost if the car is not covered during an interval of length $v$ and caught by the parking inspector, i.e.,

$$
\begin{aligned}
C(q, N) & =I(q, N)+P(q, N) \\
& =c_{I}\left[\frac{1-(1-q)^{N}}{q}\right]\left[\frac{T}{N}+\frac{1}{\lambda} \ln (1-q)\right]+c_{P}\left[1-(1-q)^{N}\right] .
\end{aligned}
$$

Since both $q$ and $N$ are decision variables, we obtain the general optimization problem as

$$
\min _{0 \leq q \leq \hat{q}, 1 \leq N \leq \hat{N}} C(q, N) .
$$

We note here that for any $N$, the expected cost function assumes the following values at the endpoints $q=0$ and $q=\hat{q}$ :

$$
\lim _{q \rightarrow 0^{+}} C(q, N)=c_{I} T, \quad \text { and } \quad C(\hat{q}, N)=c_{P}\left(1-e^{-\lambda T}\right) .
$$

Problem (5) is a mixed-integer nonlinear programme (MINLP). MINLP include mixed-integer linear programmes which are known to be $\mathcal{N} \mathcal{P}$-hard (Kannan and Monma,1978). Furthermore, MINLP include the class of quadratic integer programmes which have been proved to be undecidable (Jeroslow, 1973). To solve problem (5) we will first consider some special cases for fixed values of $N$. A general solution procedure is then proposed in Theorem 1 based on results from these special cases and structural properties of problem (5).

\subsection{Analysis of the expected total cost when $N=1$}

We first consider the case where the car owner has decided that $N=1$, i.e., that she is not willing to go out to pay the parking fees multiple times. For this case we have

$$
C(q, 1)=c_{I}\left[T+\frac{1}{\lambda} \ln (1-q)\right]+c_{P} q,
$$

so the cost function $C(q, 1)$ will equal $c_{I} T$ when $q=0$ and $c_{P}(1$ $-e^{-\lambda T}$ ) when $q=1$; see Figure 2 . We now show that the cost

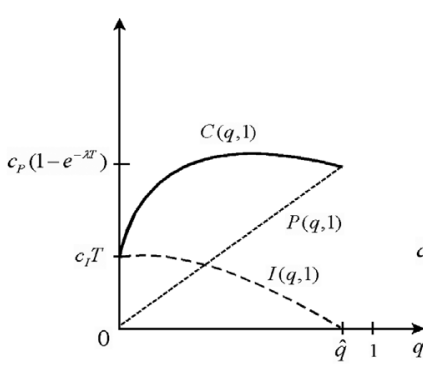

(a)

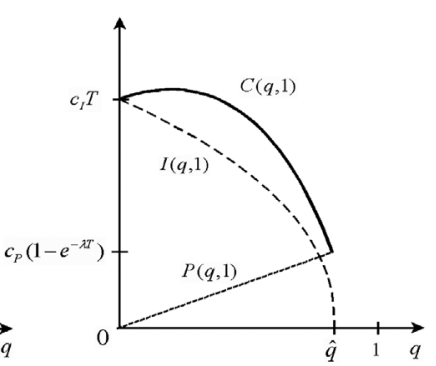

(b)
Figure 2. When $N=1$ the objective function $C(q, 1)$ is always minimized at one of the endpoints $q=0$ [graph (a)], or $q=\hat{q}$ [graph (b)].

function $C(q, 1)$ is always minimized at one of the endpoints, i.e., at point $q^{*}=0$ in Figure 2(a), or at $q^{*}=\hat{q}$ in Figure 2(b).

Proposition 2 When $N=1$, the expected cost function $C$ $(q, 1)$ is minimized at one of the endpoints.

Proof. The second derivative of $C(q, 1)$ w.r.t. to $q$ is

$$
C^{\prime \prime}(q, 1)=-\frac{c_{I}}{(1-q)^{2} \lambda}<0
$$

thus the cost function is everywhere concave and so it is impossible for the minimum cost solution to be in the interior of $[0, \hat{q}]$. This means that $C(q, 1)$ is minimized at one of the end points only, i.e., either at $q=0$, or at $q=\hat{q}$ as shown in Figure 2.

The left endpoint $q=0$ corresponds to the case where the car owner pays the full parking fee for $T$ time units at the start which gives $C(0,1)=c_{I} T$. The right endpoint $q=\hat{q}$ corresponds to the case where she does not pay anything and accepts the risk of receiving a ticket, so $C(\hat{q}, 1)=c_{P}\left(1-e^{-\lambda T}\right)$. It is now a simple matter to determine when it is optimal to pay the full amount at the outset. We find this from the condition $C(0,1)<C(\hat{q}, 1)$, or,

$$
c_{I} T<c_{P}\left(1-e^{-\lambda T}\right)
$$

We can conduct simple tests to determine under what conditions for each parameter the above inequality holds as follows:

$c_{I}$ : As long as $c_{I}<c_{P}\left(1-e^{-\lambda T}\right) / T$, it is optimal to make the full payment up-front. This is intuitive since low values of the insurance cost should result in paying the coverage cost in full.

$c_{P}$ : Similarly, as long as $c_{P}>c_{I} T /\left(1-e^{-\lambda T}\right)$, it is optimal to pay in full. This is also intuitive since high values of the penalty cost should result in paying the coverage cost in full.

$\lambda$ For this case simple algebraic manipulations imply that as long as $\lambda>-\ln \left(1-c_{I} T / c_{P}\right) / T$, it is optimal to pay in full. 
This is intuitive as a higher inspection frequency makes it more worthwhile to pay in full.

$T$ : For this parameter value, as long as $c_{P} / c_{I}>\phi(T)$ where $\phi$ $(T)=T /\left(1-e^{-\lambda T}\right)$, we must pay in full at the start. Note here that since $\lim _{T \rightarrow 0^{+}} \phi(T)=1 / \lambda$, and

$$
\phi^{\prime}(T)=\frac{1-e^{-\lambda T}-\lambda T e^{-\lambda T}}{\left(1-e^{-\lambda T}\right)^{2}}>0,
$$

there will be a unique solution to the equation $c_{P} / c_{I}=\phi(T)$ at, say, $T=\tilde{T}$ as long as $c_{P} / c_{I}>1 / \lambda$. Thus, the condition for full payment at the outset reduces to $T<\tilde{T}$, when $\tilde{T}$ exists.

\subsection{Analysis of the expected total cost when $N=2$}

When the car owner is willing to pay the parking fees at most twice, i.e., when $N=2$, the expected cost function assumes the form

$$
C(q, 2)=c_{I}(2-q)\left[\frac{T}{2}+\frac{1}{\lambda} \ln (1-q)\right]+c_{P} q(2-q) .
$$

Once again, as in the case for $N=1$, we find that when $N=2$, at the endpoints $q=0$ and $q=\hat{q}=1-e^{-\lambda T / 2}$, we have $C(0,2)=c_{I} T$, and $C(\hat{q}, 2)=c_{P}\left(1-e^{-\lambda T}\right)$. When $N=2$, the cost function $I(q, 2)$ retains its shape (i.e., it is still concave), and the cost function $P(q, 2)$ now assumes a concave form. For this reason, the optimal solution is still found at one of the endpoints as shown in the next proposition.

Proposition 3 When $N=2$, the expected cost function $C$ $(q, 2)$ is minimized at one of the endpoints.

Proof. The proof follows the same line of reasoning as in Proposition 2. Differentiating the cost function twice we find that

$$
C^{\prime \prime}(q, 2)=-\frac{c_{I} q}{(1-q)^{2} \lambda}-2 c_{P}<0 .
$$

Thus, $C(q, 2)$ is everywhere concave implying that the optimal solution must be at one of the endpoints $q=0$, or $q=\hat{q}=1-e^{-\lambda T / 2}$.

\subsection{Analysis of the expected total cost when $N \geq 3$}

When $N$ assumes values greater than two, the arguments used in Propositions 2 and 3 to prove the concavity of $C(q, N)$ no longer work because the objective function $C(q, N)$ can be nonconcave in some subinterval contained in $[0, \hat{q}]$ as shown in Example 1.

Example 1 Let $N=14$ and $\left(c_{I}, c_{P}, \lambda, T\right)=(4,8,1,2)$. It follows that $\hat{q}=0.1331$. Then it can be shown that $C^{\prime \prime}(q, 14)$ is negative in $[0,0.1305]$ and positive in $[0.1305, \hat{q}]$, implying that the function is nonconcave in the latter interval.
In Propositions 4 and 5 we obtain simple sufficient conditions which assure that the cost function is minimized at the endpoint $q=\hat{q}$ and $q=0$, respectively, for any value of $N$.

Proposition 4 A sufficient condition for the cost function $C$ $(q, N)$ to be minimized at the right endpoint $q=\hat{q}$ for any positive value of $N$ is $c_{P} / c_{I}<1 / \lambda$.

Proof. To prove this, let's first write the expected cost function (4) in the equivalent form

$$
C(q, N)=\left[\frac{1-(1-q)^{N}}{q}\right]\left\{c_{I}\left[\frac{T}{N}+\frac{1}{\lambda} \ln (1-q)\right]+c_{P} q\right\},
$$

or, $C(q, N)=\kappa(q, N) \ell(q, N)$, where, as before, $\kappa(q, N)=[1-(1$ $\left.-q)^{N}\right] / q$, and $\ell(q, N)=c_{I}[T / N+\ln (1-q) / \lambda]+c_{P} q$. Differentiating $C$ $(q, N)$ w.r.t. $q$, we have $C^{\prime}(q, N)=\kappa \ell^{\prime}+\kappa^{\prime} \ell$ where

$$
\ell^{\prime}=c_{P}-\frac{c_{I}}{(1-q) \lambda} .
$$

Now, since $\kappa^{\prime}<0$ and $\ell>0$, we always have $\kappa^{\prime} \ell<0$. But, since $\kappa>0$, provided that $\ell^{\prime}<0$, or

$$
\frac{c_{P}}{c_{I}}<\frac{1}{(1-q) \lambda},
$$

we have $\kappa \ell^{\prime}<0$, and thus $C^{\prime}(q, N)<0$. Note that the righthand-side of (6) starts at $1 / \lambda$ and approaches infinity as $q$ approaches 1 . Thus, whenever $c_{P} / c_{I}<1 / \lambda$, the condition (6) is also satisfied which results in a decreasing function $C(q, N)$ with the minimum at $q^{*}=\hat{q}$.

Remark 3 It is useful to emphasize that the condition given in Proposition 4 is sufficient meaning that there may be (in fact, there are) parameter value combinations other than $c_{P} / c_{I}<1 / \lambda$ for which the optimal solution is still at the endpoint $q=\hat{q}$.

We now show that under some fairly easy-to-calculate conditions the cost function is minimized at $q=0$.

Proposition 5 A sufficient condition for the cost function $C$ $(q, N)$ to be minimized at the left endpoint $q=0$ for any positive value of $N$ is

$$
\frac{c_{P}}{c_{I}}>\max \left\{\frac{1}{\lambda}+\frac{1}{2}\left(1-\frac{1}{N}\right) T, \frac{1-e^{-\lambda T}}{\lambda N\left(1-e^{-\lambda T / N}\right) e^{-\lambda T}}\right\} .
$$

Proof. Differentiating the cost function $C(q, N)$ we have $C^{\prime}$ $(q, N)=I^{\prime}(q, N)+P^{\prime}(q, N)$. In order for the cost function to increase for all $q \in[0, \hat{q}]$, we must have, first of all, that $P^{\prime}(0, N)>$ $-I^{\prime}(0, N)$. From (3) we have $P^{\prime}(0, N)=c_{P} N$ and from (2), we obtain $I^{\prime}(0, N)=c_{I}\left[\frac{1}{2}(1-N) T-N / \lambda\right] \quad$ since $\quad \kappa^{\prime}(0, N)=$ $\frac{1}{2} N(1-N), u(0, N)=T / N, \kappa(0, N)=N$ and $u^{\prime}(0, N)=-1 / \lambda$. Simplifying the condition $P^{\prime}(0, N)>-I^{\prime}(0, N)$ gives

$$
\frac{c_{P}}{c_{I}}>\frac{1}{\lambda}+\frac{1}{2}\left(1-\frac{1}{N}\right) T .
$$

In order for the cost function to increase for all $q \in[0, \hat{q}]$, we must also have $P^{\prime}(\hat{q}, N)>-I^{\prime}(\hat{q}, N)$. We now note from 
Figure 3 that if at both endpoints $P^{\prime}(\cdot, N)$ is higher than $-I^{\prime}(\cdot, N)$, i.e., $\left[P^{\prime}(0, N)>-I^{\prime}(0, N)\right.$ and $\left.P^{\prime}(\hat{q}, N)>-I^{\prime}(\hat{q}, N)\right]$, then it is necessary for $P^{\prime}(q, N)$ and $-I^{\prime}(q, N)$ to intersect an even number of times (that is, twice, four times, etc.) which results in multiple local optima. But given the nature of $I(q, N)$ which is decreasing, and $P(q, N)$ which is increasing concave, as shown in Section 3.2, multiple local optima cannot occur. Since $P^{\prime}(\hat{q}, N)>-I^{\prime}(\hat{q}, N)$ simplifies to

$$
\frac{c_{P}}{c_{I}}>\frac{1-e^{-\lambda T}}{\lambda N\left(1-e^{-\lambda T / N}\right) e^{-\lambda T}},
$$

the condition (7) assures that $C(q, N)$ is monotone increasing and guarantees the optimality of the endpoint $q=0$.

\subsection{A general Solution Procedure}

In the above analysis we have established conditions under which the optimal value of $q$ assumes one of its extreme points,

$$
\left(u^{*}, v^{*}\right)=\left\{\begin{array}{l}
\left(\frac{T}{N^{*}}, 0\right) \\
\left(0, \frac{T}{N^{*}}\right) \\
\left(\frac{T}{N^{*}}+\frac{\ln \left(1-\tilde{q}\left(N^{*}\right)\right)}{\lambda},-\frac{\ln \left(1-\tilde{q}\left(N^{*}\right)\right)}{\lambda}\right)
\end{array}\right.
$$

(a) If $\frac{c_{P}}{c_{I}}>R_{U}$ then

$N^{*}$ is an arbitrary integer number in $[1, \hat{N}]$

and

$$
\begin{aligned}
& \left(u^{*}, v^{*}\right)=\left(\frac{T}{N^{*}}, 0\right), \text { where } \\
& R_{U}=\max \left\{\frac{T}{1-e^{-\lambda T}}, \frac{1}{\lambda}+\frac{1}{2}\left(1-\frac{1}{N^{*}}\right) T, \frac{1-e^{-\lambda T}}{\lambda N^{*}\left(1-e^{-/ N^{*}}\right) e^{-\lambda T}}\right\} .
\end{aligned}
$$

(b) If $\frac{c_{P}}{c_{I}}<R_{L}$ then

$N^{*}$ is an arbitrary integer number in $[1, \hat{N}]$

and

$$
\begin{aligned}
& \left(u^{*}, v^{*}\right)=\left(0, \frac{T}{N^{*}}\right), \\
& \text { where } R_{L}=\min \left\{\frac{1}{\lambda}, \frac{T}{1-e^{-\lambda T}}\right\} .
\end{aligned}
$$

(c) If $\frac{c_{P}}{c_{I}} \in\left[R_{L}, R_{U}\right]$ then conduct an exhaustive search over all values of $N$ to find

$N^{*}=\min _{1 \leq N \leq \hat{N}} C(\tilde{q}(N), N)$,

where $\tilde{q}(N)$ is such that $\left.\frac{d C(q, N)}{d q}\right|_{q=\tilde{q}(N)}=0$ for each integer number in $[1, \hat{N}]$, and

$$
\begin{aligned}
& \text { if } \frac{d^{2} C\left(\tilde{q}\left(N^{*}\right), N^{*}\right)}{d q^{2}} \leq 0 \text { and } \frac{c_{P}}{c_{I}} \geq \frac{T}{1-e^{-\lambda T}} \\
& \text { if } \frac{d^{2} C\left(\tilde{q}\left(N^{*}\right), N^{*}\right)}{d q^{2}} \leq 0 \text { and } \frac{c_{P}}{c_{I}}<\frac{T}{1-e^{-\lambda T}} \\
& \text { if } \frac{d^{2} C\left(\tilde{q}\left(N^{*}\right), N^{*}\right)}{d q^{2}}>0 .
\end{aligned}
$$

either 0 or $\hat{q}$. In Theorem 1 we address the problem of jointly solving for $q$, or alternatively $u$ and $v$, and $N$.

Theorem 1 The optimal solution to the parking problem (5) is to visit the parking space $N^{*}$ times and each time pay for $u^{*}$ units of time and leave $v^{*}$ units of uncovered time, where $\left(N^{*}\right.$, $\left.u^{*}, v^{*}\right)$ are found as follows:

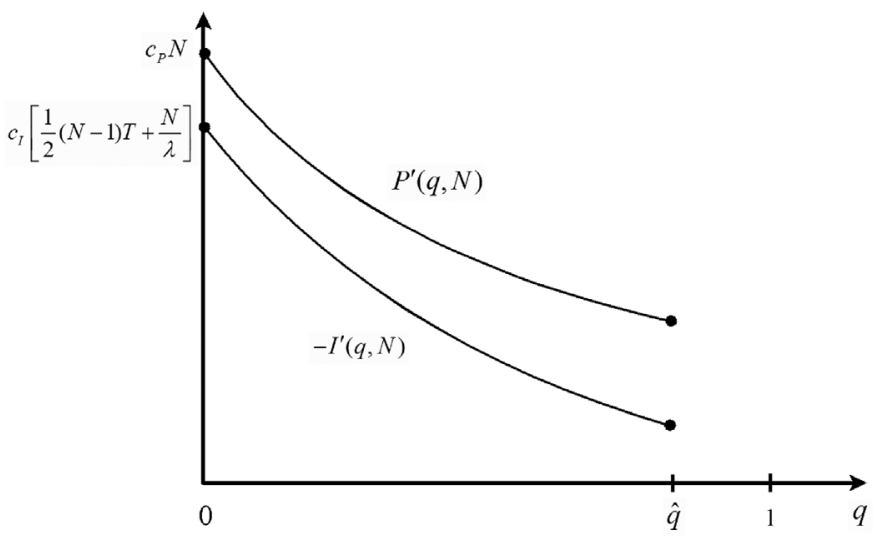

Figure 3. When $P^{\prime}(q, N)>-I^{\prime}(q, N)$ for all $q \in[0, \hat{q}]$, the cost function is always increasing, and thus $q^{*}=0$.
Proof. First we note that the extreme values 0 and $\hat{q}$ of $q^{*}$ correspond to values of $\left(\frac{T}{N}, 0\right)$ and $\left(0, \frac{T}{N}\right)$ for $\left(u^{*}, v^{*}\right)$, respectively. Furthermore, note that $\lim _{q \rightarrow 0^{+}} C(q, N)=c_{I} T$, and $C(\hat{q}, N)=c_{P}\left(1-e^{-\lambda T}\right)$.

Now, to prove Part (a) note that from Propositions 2, 3 and 5 we conclude that $q^{*}$ takes one of its extreme values, 0 or $\hat{q}$. However, since $\frac{c_{P}}{c_{I}}>R_{U} \geq \frac{T}{1-e^{-i T}}$, it follows that $c_{I} T<c_{P}(1$ $-e^{-\lambda T}$ ) and therefore $q^{*}=0$ implying that $v^{*}=0, u^{*}=\frac{T}{N}$ and $N^{*}$ can take any arbitrary integer value in $[1, \hat{N}]$.

For Part (b), similarly we use the results of Propositions 2, 3 and 4 to conclude that $q^{*}$ takes one of its extreme values, 0 or $\hat{q}$. However, since $\frac{c_{P}}{c_{I}}<R_{L} \leq \frac{T}{1-e^{-\lambda T}}$, it follows that $c_{I} T>c_{P}(1$ $-e^{-\lambda T}$ ) and therefore $q^{*}=\hat{q}$ implying that $v^{*}=\frac{T}{N}, u^{*}=0$ and $N^{*}$ can take any arbitrary integer value in $[1, \hat{N}]$.

Finally, Part (c) addresses the case where none of the conditions of Propositions 4 and 5 hold. In such a case, we perform an exhaustive search on $N \in[1, \hat{N}]$. For each $N$ we find $\tilde{q}(N)$ that solves the first order conditions for $C(q(N), N)$. Note that there is a unique value for $\tilde{q}(N)$ given that $C(q, N)$ is formed by two functions one of which is decreasing (Proposition 1) and the other increasing concave (Lemma 4). Once $\tilde{q}(N)$ is calculated we check whether it corresponds to a maximum or minimum point. If it ia a maximum point, i.e., $\frac{d^{2} C\left(\tilde{q}\left(N^{*}\right), N^{*}\right)}{d q^{2}} \leq 0$ then $q^{*}$ 
takes one of its extreme values, 0 or $\hat{q}$, depending on the value of the ratio $\frac{c_{P}}{c_{I}}$. If it is a minimum point, i.e., $\frac{d^{2} C\left(\tilde{q}\left(N^{*}\right), N^{*}\right)}{d q^{2}}>0$, then $q^{*}$ will have an interior optimum value $\tilde{q}(N)$ and the optimal values of $u^{*}$ and $v^{*}$ are calculated based on the knowledge that $q=1-e^{-\lambda v}$ and $u+v=\frac{T}{N}$.

Remark 4 Although in Parts (a) and (b) of Theorem 1 we find that $N^{*}$ can be any arbitary integer in $[1, \hat{N}]$, in practice $N=1$ would suffice. Such a solution would, for example, make sense when there is a setup cost associated with visiting the parking space. This issue is explored in more details in Section 6.

\section{NUMERICAL EXAMPLES}

In this section we consider numerical examples for $N=1,2,3$ and 4 and examine the effect of varying the parameter values on the optimal solution.

\subsection{Optimal solution at the left endpoint $q^{*}=0$}

First consider the case with $N=4$ and $\left(c_{I}, c_{P}, \lambda, T\right)=(\$ 1 /$ hour, $\$ 8,1 /$ hour,2 hours). For these data values, the first derivative of the objective function is $C^{\prime}(q, 4)=a(q) / b(q)$ where,

$$
\begin{aligned}
a(q)= & 64 q^{4}+[6 \ln (1-q)-251] q^{3} \\
& +[365-22 \ln (1-q)] q^{2}+[28 \ln (1-q)-230] q \\
& +[50-12 \ln (1-q)] \\
b(q)= & 2(1-q)>0 .
\end{aligned}
$$

For this problem, we have

$$
\begin{aligned}
\frac{c_{P}}{c_{I}} & =8, \quad \frac{1}{\lambda}+\frac{1}{2}\left(1-\frac{1}{N}\right) T=1.75, \\
\frac{T}{1-e^{-\lambda T}} & =2.3, \quad \text { and } \frac{1-e^{-\lambda T}}{\lambda N\left(1-e^{-\lambda T / N}\right) e^{-\lambda T}}=4.06,
\end{aligned}
$$

and thus condition (7) of Proposition 5 shows that the optimal solution is at the boundary point $q^{*}=0$ which implies $v=0$, and $u=L=T / N=\frac{1}{2}$. In other words, the car owner should pay the full parking fee for each of the periods of length $L=\frac{1}{2}$ at the beginning of the period; see Figure 4. This is intuitive since the insurance $\operatorname{cost} c_{I}=$ /hour is much lower than the maximum penalty $\operatorname{cost} c_{P}=$ during the duration of stay $T=2$ hours. Therefore it makes sense to pay in full at the beginning.

Until now we have assumed that the car owner has decided what the value of $N$ is and have solved the problems with the given value of $N$. But this value, i.e., the number of times the car owner is willing to return to her car to pay the parking fees is a decision variable; so we must find its optimal value along with $q$.

If we reconsider the problem above with the parameter values $\left(c_{I}, c_{P}, \lambda, T\right)=(1,8,1,2)$ but make $N$ a decision variable and plot the total expected cost for $N=1, \ldots, 4$, we find from Figure 5 that the optimal solution is now to set $q^{*}=0$ as before,

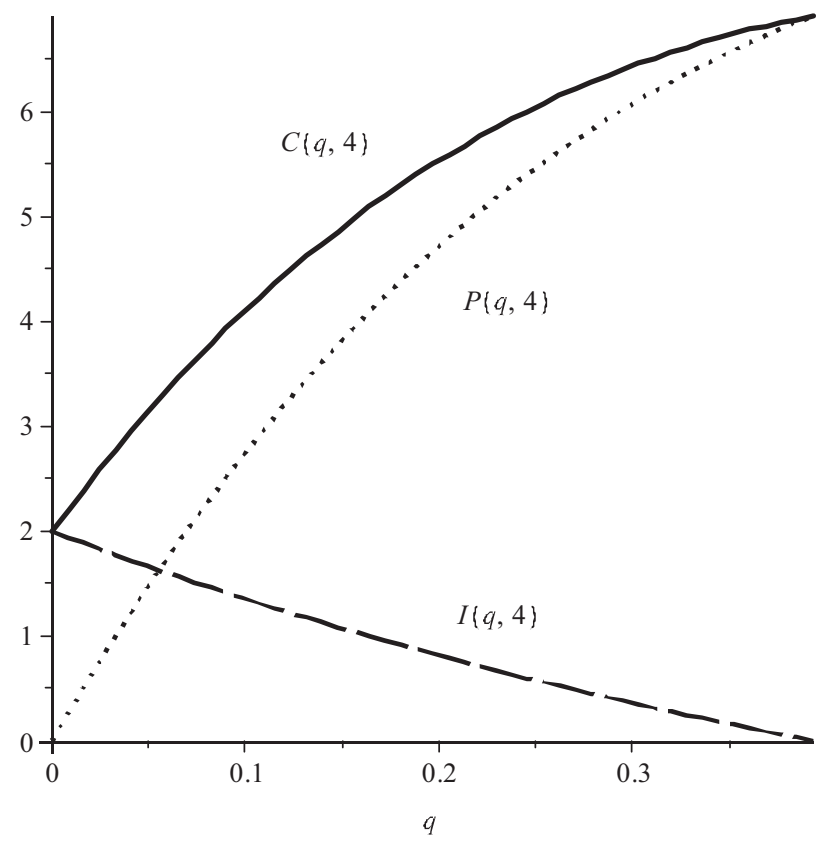

Figure 4. The cost function $C(q, 4)$ with $\left(c_{I}, c_{P}, \lambda, T\right)=(1,8,1,2)$ is monotone increasing over $[0, \hat{q}]$, so the optimal solution is to pay for coverage in full at the beginning, i.e., $q=v=0$, and $u=L$.

and the optimal $N^{*}$ is found to be an arbitrary value. If there is any loss of convenience associated with returning to the car, then the car owner should pay the full cost of parking initially for $T=2$ hours and not bother checking her car at periodic intervals, i.e., $N=1$.

\subsection{Optimal solution at the right endpoint $q^{*}=\hat{q}$}

Next, consider again the case with $N=4$ and $\left(c_{I}, c_{P}, \lambda, T\right)=$ $(4,8,1,2)$. Here, since the insurance cost $c_{I}$ is four times as high as it was before, and the other parameters are the same, one would expect the patron to exhibit "risky" behaviour and not pay for parking at all. For this case, the first derivative is $C^{\prime}(q, 4)$ $=a(q) / b(q)$, where

$$
\begin{aligned}
\frac{1}{2} a(q)= & 16 q^{4}+[6 \ln (1-q)-59] q^{3} \\
& +[77-22 \ln (1-q)] q^{2}+[28 \ln (1-q)-38] q \\
& +[2-12 \ln (1-q)] \\
b(q)= & 1-q>0 .
\end{aligned}
$$

Solving $C^{\prime}(q, 4)=0$ gives the stationary point at $\tilde{q}=0.09$, thus unlike the previous case the cost function is not monotone. To check whether this point is a minimum or maximum, we 


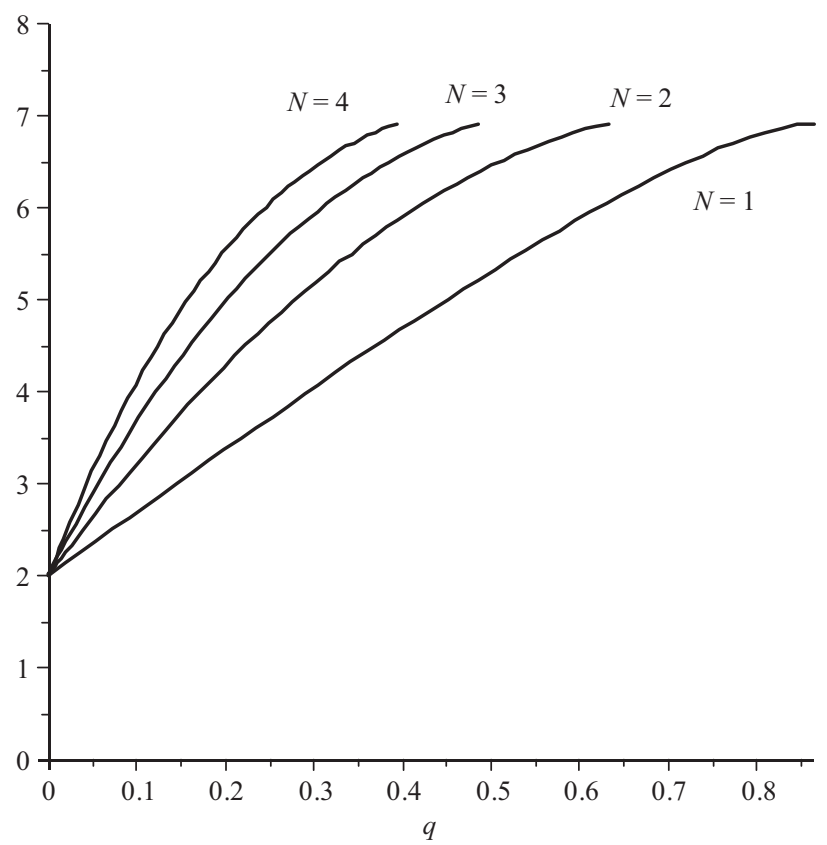

Figure 5. The expected cost functions $C(q, N)$ for $N=1, \ldots, 4$. Here, the optimal $q^{*}=0$, but the optimal value of $N$ is arbitrary when $\left(c_{l}, c_{P}, \lambda, T\right)=(1,8,1,2)$.

compute the second derivative as $C^{\prime \prime}(q, 4)=\alpha(q) / \beta(q)$, where

$$
\begin{aligned}
\alpha(q)= & -94 q^{4}+[352-24 \ln (1-q)] q^{3} \\
& +[80 \ln (1-q)-464] q^{2}+[252-88 \ln (1-q)] q \\
& +[32 \ln (1-q)-48] \\
\beta(q)= & (1-q)^{2}>0 .
\end{aligned}
$$

Now, substituting the stationary point $\tilde{q}=0.09$ into the second derivative, we find $C^{\prime \prime}(\tilde{q}, 4)=-37.18$ implying that the function reaches its maximum at this point. Thus, the optimal solution must still be at an endpoint. In this case since $\hat{q}=1-e^{-\lambda T / N}=0.39$, we have $C(0,4)=8$, and $C(\hat{q}, 4)=6.91$, so it is optimal to choose $q^{*}=\hat{q}=0.39$ and not pay for parking; a financially optimal, but certainly unethical behaviour. See Figure 6 for a graph of the objective function with the local maximum at $\tilde{q}=0.09$.

Note that with $\left(c_{I}, c_{P}, \lambda, T\right)=(4,8,1,2)$, the sufficient condition provided in Proposition 4 is not helpful in determining whether or not $q^{*}=\hat{q}$. If, however, we revise the data as $\left(c_{I}, c_{P}, \lambda, T\right)=$ $(4,8,0.1,2)$, we obtain $c_{P} / c_{I}=2$, and $1 / \lambda=10$ in which case we can employ Proposition 4 to show that the optimal solution is at the endpoint $q^{*}=\hat{q}$.

If we again reconsider the problem above with the parameter values $\left(c_{1}, c_{P}, \lambda, T\right)=(4,8,1,2)$ but make $N$ a decision variable and plot the total expected cost for $N=1, \ldots, 4$, we find from Figure 7 that the optimal solution is still to set $q^{*}=\hat{q}$ as before for

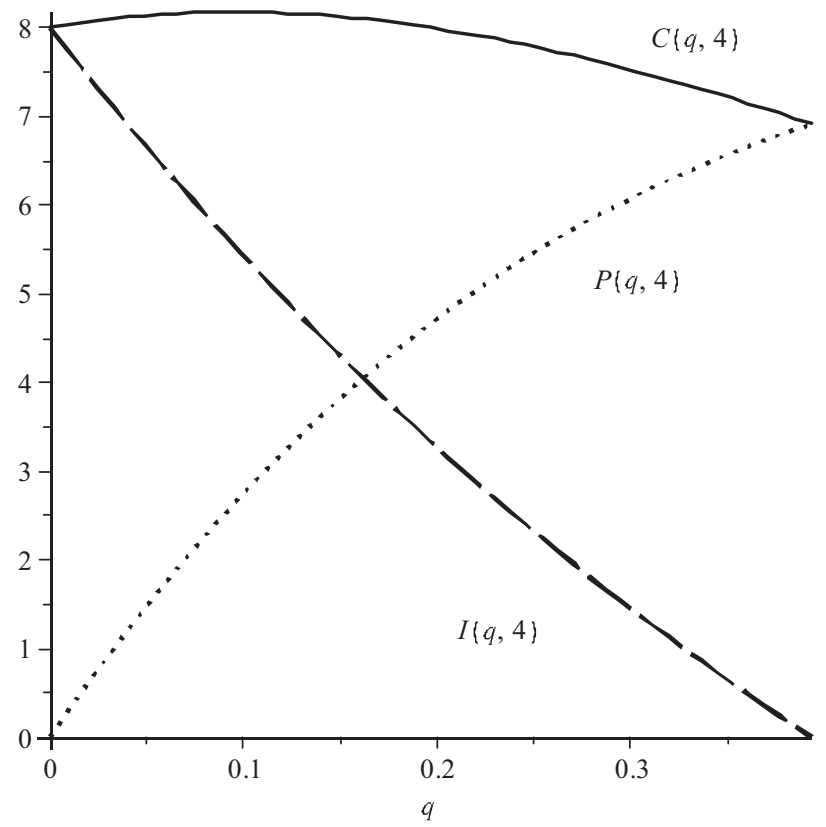

Figure 6. The objective function $C(q, 4)$ with $\left(c_{I}, c_{P}, \lambda, T\right)=(4,8,1,2)$ is not monotone, i.e., it has a maximum at $\tilde{q}=0.09$. This objective function is minimized at the endpoint $q^{*}=\hat{q}=0.39$.

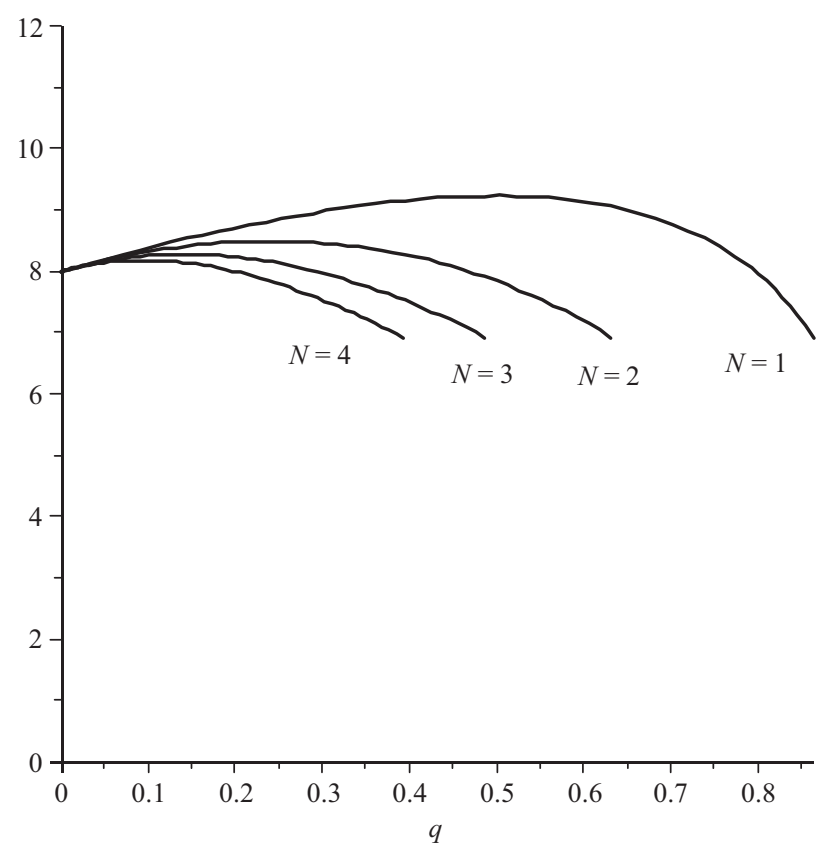

Figure 7. The expected cost functions $C(q, N)$ for $N=1, \ldots, 4$. Here, it is optimal to set $q^{*}=\hat{q}$ when $\left(c_{I}, c_{P}, \lambda, T\right)=(4,8,1,2)$ for any value of $N$. 
all values of $N=1, \ldots, 4$, and the optimal $N^{*}$ is found to be an arbitrary value. Since it is optimal not to pay at all, it makes sense for the car owner not to bother checking her car at periodic intervals, thus, as in the previous example, $N$ can be set to 1 .

\subsection{Sensitivity analysis}

To analyze numerically the effect of varying parameters on the optimal solution, we start with a set of base values $\left(c_{I}, c_{P}, \lambda, T, N\right)$ $=(1,8,1,2,4)$. We then vary these parameter values and record the changes (if any) in the optimal solution. For the base parameters, as we had seen in Section 5.1, the optimal solution is found as $q^{*}=v^{*}=0$, so that $u^{*}=L=\frac{1}{2}$, that is, the car owner should pay the full parking fee in each subinterval of length $\frac{1}{2}$. Table 1 summarizes the results of sensitivity analysis.

For $c_{I}=\left\{\frac{1}{4}, \frac{1}{2}, 1,2\right\}$, the solution does not change, i.e., $q^{*}=$ 0 and $C(0, N)<C(\hat{q}, N)$. However, when this cost parameter increases to $c_{I}=4$, the optimal solution moves to the other endpoint $q^{*}=\hat{q}=0.39$ where we find $C(0, N)=8$ and $C(\hat{q}, N)=$ 6.91. Such an analysis could be used by parking services to study the effect of parking fees on drivers behaviour. In this example we see that we have to significantly increase the fees in order to see a change in the behaviour of the driver.

Similarly, when $c_{P}=\{4,8,16,32\}$, the optimal solution is still as before, i.e., $q^{*}=0$ with $C(0, N)=2$. But, when $c_{P}$ is reduced substantially to $c_{P}=2$, it becomes optimal to take the risk and not pay for parking, that is, $q^{*}=\hat{q}=0.39$ with $C(\hat{q}, N)=1.72<2$. We observe that the fine plays an important role in enforcing parking regulations. This is consistent with the study of Elliot and Wright (1982), Fishman and Miguel (2007), and Petiot (2004).

For $\lambda$ the results are similar to those we have found for $c_{P}$, that is, only for relatively small values of $\lambda$ it is optimal not to pay for parking since in this case it is unlikely that the parking inspector will ticket the car. This is an interesting finding, as it links the frequency of parking inspector patrols to magnitude of the fine. Parking services could use this trade-off to align the patrol frequency with that of the fine. On the other hand, for $T$, the results are similar to those we have found for $c_{I}$, that is, for relatively large values of $T$ it makes sense to pay for parking in full. This is also intuitive since large values of $T$ make it more likely for the parking inspector to ticket the car if the car owner does not pay for parking.

\section{SOME EXTENSIONS}

In this section we briefly describe two extensions. In the first extension, we incorporate the setup cost that may be incurred if the car owner has to return to the parking space after every $L$ time units to pay the parking fees. In the second extension, we consider the case of random $T$.

\subsection{Expected cost of setup $S(q, N)$}

To evaluate this cost we define a new random variable $M$ defined as the number of times the car owner returns to the parking space to pay the parking fees, if necessary. Thus, $M$ assumes the values $1,2, \ldots, N-1$ for $N \geq 2$. For example, when $N=4$, we have $M=1$ if the car is ticketed during $v$ in the first interval; the car owner returns to the parking space and finds that she has a ticket with probability $g(1)=q$. Since a ticket has been received, she keeps the car parked in its current spot and returns to the parking space only at $T$ to drive away. We have $M=2$ if no ticket has been received in period one, but it has been received in period two with probability $g(2)=(1-q) q$. Finally, $M=3$ with probability $g(3)=(1-q)^{2} \cdot 1$ if the car owner has not received a ticket during the first two exposed intervals (regardless of what happened in the very last interval); see Figure 8.

In general, then, defining $g(m)$ as the p.m.f. of $M$, we have

$$
\begin{aligned}
g(m) & =(1-q)^{m-1} q, \quad m=1, \cdots, N-2 \\
g(N-1) & =(1-q)^{N-2} .
\end{aligned}
$$

Since $g(m)$ is a p.m.f., we have $\sum_{m=1}^{N-1} g(m)=1$ and the expected value of $M$ is

$$
\gamma(q, N) \equiv E(M)=\left\{\begin{array}{ll}
0, & \text { for } N=1 \\
N-1] / q, & \text { for } N \geq 2
\end{array},\right.
$$

which has properties very similar to those of $\kappa(q, N)$, i.e., $\lim _{q} \rightarrow$ $0^{+} \gamma(q, N)=N-1$ and that $\gamma(q, N)$ is decreasing in $q$.

Now, if we define $c_{S}$ as the cost of setup, then the expected cost of setup during $T$ is simply

$$
S(q, N)=c_{S} \gamma(q, N) .
$$

Incorporating this cost term into the overall expected cost, we obtain

$$
\begin{aligned}
C(q, N)= & I(q, N)+S(q, N)+P(q, N) \\
= & c_{I}\left[\frac{1-(1-q)^{N}}{q}\right]\left[\frac{T}{N}+\frac{1}{\lambda} \ln (1-q)\right] \\
& +c_{S}\left[\frac{1-(1-q)^{N-1}}{q}\right]+c_{P}\left[1-(1-q)^{N}\right] .
\end{aligned}
$$

It is interesting to note that, when $N=2$, the setup cost assumes the form $S(q, 2)=c_{S}$, a constant. Thus, the optimal policy found in Proposition 3 does not change, i.e., one still finds the solution either at $q=0$, or at $q=\hat{q}$.

Let us now reconsider the case in Section 5.2 with $\left(c_{I}, c_{P}, \lambda\right.$, $T)=(4,8,1,2)$ and $c_{S}=2.5$, but allow $N$ to be a decision variable. In this case the optimal solution is obtained as $q^{*}=\hat{q}$ (as before), but we find that due to the costliness of returning to the car, we find optimal $N^{*}=1$ as we see in Figure 9.

\subsection{Random $T$}

In the analysis presented so far, we assumed that the car owner will need to park her car for a total, and deterministic, duration of $T$ hours. Naturally, in some cases this may not be a realistic 
TABLE 1.

Sensitivity analysis of the optimal solution for varying parameter values. In this table the rows indicated by bold font correspond to the right endpoint optimal solution $q^{*}=\hat{q}$, (i.e., $v^{*}=L$ ) and the remaining rows to the left endpoint solution $q^{*}=0$ (i.e., $v^{*}=0$ ).

\begin{tabular}{|c|c|c|c|c|c|c|c|c|c|c|c|}
\hline$c_{I}$ & $c_{P}$ & $\lambda$ & $T$ & $N$ & $q^{*}$ & $u^{*}$ & $v^{*}$ & $C\left(q^{*}, N\right)$ & $\hat{q}$ & $C(\mathbf{0}, N)$ & $C(\hat{\boldsymbol{q}}, \boldsymbol{N})$ \\
\hline$\frac{1}{4}$ & & & & & 0 & $\frac{1}{2}$ & 0 & $\frac{1}{2}$ & 0.39 & $\frac{1}{2}$ & 6.91 \\
\hline$\frac{1}{2}$ & & & & & 0 & $\frac{1}{2}$ & 0 & 1 & 0.39 & 1 & 6.91 \\
\hline 1 & 8 & 1 & 2 & 4 & 0 & $\frac{1}{2}$ & 0 & 2 & 0.39 & 2 & 6.91 \\
\hline 2 & & & & & 0 & $\frac{1}{2}$ & 0 & 4 & 0.39 & 4 & 6.91 \\
\hline \multirow[t]{2}{*}{4} & & & & & 0.39 & $\mathbf{0}$ & $\frac{1}{2}$ & 6.91 & 0.39 & 8 & 6.91 \\
\hline & 4 & & & & 0 & $\frac{1}{2}$ & 0 & 2 & 0.39 & 2 & 3.45 \\
\hline \multirow[t]{4}{*}{1} & 8 & 1 & 2 & 4 & 0 & $\frac{1}{2}$ & 0 & 2 & 0.39 & 2 & 6.91 \\
\hline & 16 & & & & 0 & $\frac{1}{2}$ & 0 & 2 & 0.39 & 2 & 13.83 \\
\hline & 32 & & & & 0 & $\frac{1}{2}$ & 0 & 2 & 0.39 & 2 & 27.66 \\
\hline & & $\frac{1}{8}$ & & & 0.06 & $\mathbf{0}$ & $\frac{1}{2}$ & 1.76 & 0.06 & 2 & 1.76 \\
\hline & & 2 & & & 0 & $\frac{1}{2}$ & 0 & 2 & 0.63 & 2 & 7.85 \\
\hline & & 4 & & & 0 & $\frac{1}{2}$ & 0 & 2 & 0.86 & 2 & 7.99 \\
\hline & & & $\frac{1}{2}$ & & 0 & $\frac{1}{8}$ & 0 & $\frac{1}{2}$ & 0.11 & $\frac{1}{2}$ & 3.14 \\
\hline & & & 1 & & 0 & $\frac{1}{4}$ & 0 & 1 & 0.22 & 1 & 5.05 \\
\hline \multirow[t]{5}{*}{1} & 8 & 1 & 2 & 4 & 0 & $\frac{1}{2}$ & 0 & 2 & 0.39 & 2 & 6.91 \\
\hline & & & 3 & & 0 & $\frac{3}{4}$ & 0 & 3 & 0.52 & 3 & 7.60 \\
\hline & & & 4 & & 0 & 1 & 0 & 4 & 0.63 & 4 & 7.85 \\
\hline & & & 10 & & 0.91 & $\mathbf{0}$ & 2.5 & 7.99 & 0.91 & 10 & 7.99 \\
\hline & & & & 1 & 0 & 2 & 0 & 2 & 0.86 & 2 & 6.91 \\
\hline
\end{tabular}

assumption as it may be difficult to know when one's business will be completed. (Think of visiting a doctor's office.) In such a case one can formulate the problem assuming that the duration $T$ itself is a random variable and solve the problem accordingly. To illustrate, using the results obtained for the deterministic $T$ model discussed above, let us assume that we want to compare the expected costs of paying in full and not paying at all, denoted in this case by $\bar{C}(0,1)$ and $\bar{C}(\hat{q}, 1)$, respectively. If $T$ has a p.d.f. $h(t)$, then it follows that

$$
\begin{aligned}
& \bar{C}(0,1)=c_{I} E(T)=c_{I} \int_{0}^{\infty} \operatorname{th}(t) d t, \\
& \bar{C}(\hat{q}, 1)=c_{P} E\left(1-e^{-\lambda T}\right)=c_{P} \int_{0}^{\infty}\left(1-e^{-\lambda t}\right) h(t) d t .
\end{aligned}
$$

Assuming that $T$ is exponential with mean $1 / \mu$ and rate $\mu$, i.e., with p.d.f. $h(t)=\mu e^{-\mu t}$, then

$$
\bar{C}(0,1)=\frac{c_{I}}{\mu}, \text { and } \quad \bar{C}(\hat{q}, 1)=\frac{c_{P} \lambda}{\lambda+\mu} .
$$

Thus, we see that $\bar{C}(0,1)>\bar{C}(\hat{q}, 1)$, (i.e., $q^{*}=\hat{q}$, so never pay), if and only if

$$
\frac{c_{P}}{c_{I}}<\frac{1}{\lambda}\left(1+\frac{\lambda}{\mu}\right)
$$

We recall from Proposition 4 that it is optimal to choose $q^{*}=\hat{q}$ when $c_{P} / c_{I}<1 / \lambda$. Comparing this to the above result (8), we see that if the length of time $T$ is exponential with mean 

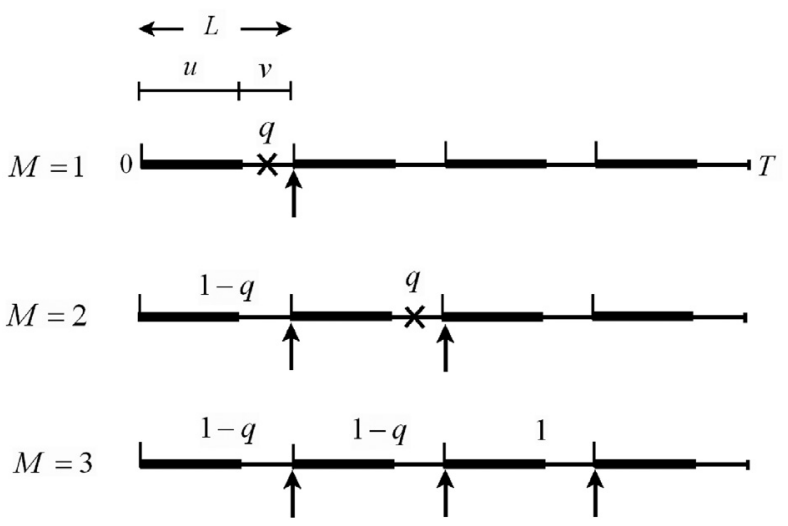

Figure 8. The random variable $M$ defined as the number of times the car owner returns to the parking space to pay the parking fees, if necessary.

$1 / \mu$, then the car owner can be somewhat less risk averse and decide not to pay at all for a wider range of $c_{P} / c_{I}$ values. This result follows since for an exponential random variable $T$, we have $\operatorname{Pr}$ $(T \leq 1 / \mu)=1-e^{-1} \simeq 0.63$, i.e., there is a higher probability that $T$ will end before its mean value.

\section{SUMMARY}

In this paper, we have considered a practical problem that arises in parking spaces. Assuming that the car owner needs to park her car for $T$ time units, and that she is willing to return to the parking space at most $N$ times to pay the parking fees, what is the optimal duration of coverage during a subinterval of length $T / N$ ? We have shown that under the assumption of Poisson arrivals of the parking inspector, the optimal policy is either to pay in full or to pay nothing when $N=1,2$. For higher values of $N$, we have presented sufficient conditions, under which it is optimal to pay in full or to pay nothing. We also discussed two extensions to the basic model, where, (i) a setup cost is incurred each time a parking fee is paid, and (ii) the duration of stay is random.

An important assumption in our model was the exponential interarrival times of the parking inspector. We suspect that if this assumption is relaxed and we allow for more general interarrival times, the results will be different. We leave this investigation for future studies of this problem.

Throughout the paper we assumed that the total period $T$ is divided into subintervals of equal length and that in each subinterval the duration of covered and uncovered periods are the same. A further generalization of the model would involve relaxing this assumption and allowing for different subinterval lengths and different covered/uncovered intervals within each subinterval. Such a generalization would require using dynamic programming and may reveal further insights into the problem.

Finally, an interesting extension would be to consider the value of money invested in the insurance coverage. This is especially relevant in the insurance context where the annual premium may be a significant investment.

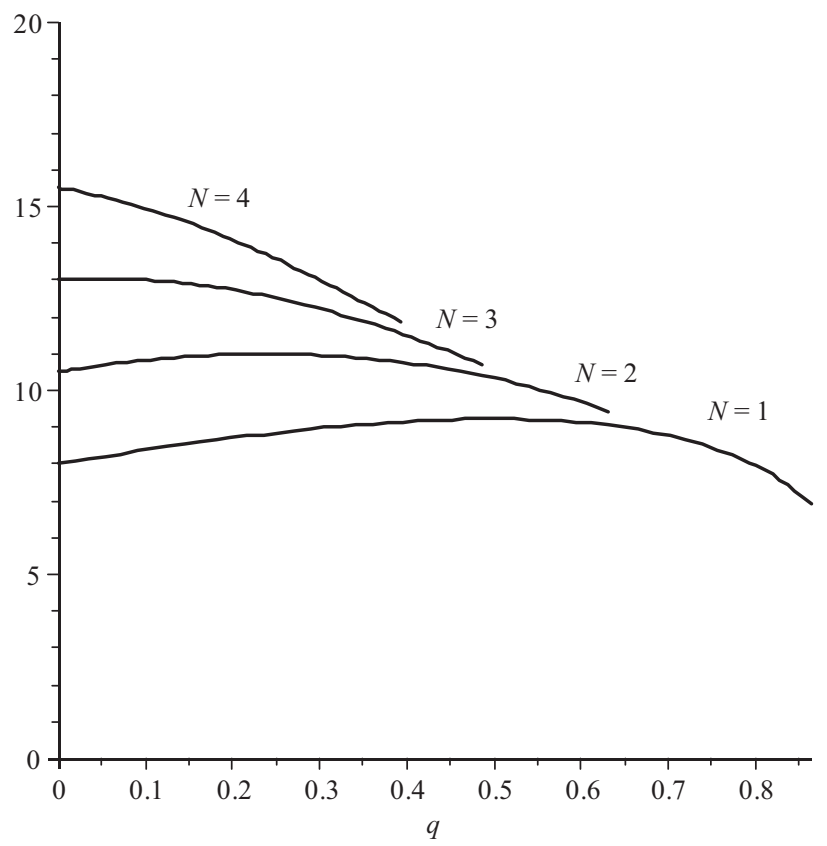

Figure 9. The expected cost functions $C(q, N)$ for $N=1, \ldots, 4$. Here, the optimal solution with $\left(c_{l}, c_{P}, \lambda, T\right)=(4,8,1,2)$ and $c_{S}=2.5$ is still $q^{*}=\hat{q}$, but $N^{*}=$ 1. 


\section{Acknowledgments}

We would like to thank the anonymous reviewers and an associate editor for their valuable comments and suggestions. The last two authors acknowledge financial support from The Natural Sciences and Engineering Research Council through their Discovery Grants Program.

\section{REFERENCES}

Arnott, R., and Inci, E. (2006), "An integrated model of downtown parking and traffic congestion," Journal of Urban Economics, 60(3): 418-442.

Arnott, R., and Rowse, J. (1999), "Modeling parking," Journal of Urban Economics, 45(1): 97-124.

Arnott, R., and Rowse, J. (2009), "Downtown parking in auto city," Regional Science and Urban Economics, 39(1): 1-14.

Cassady C.R., and Kobza, J.E. (1998), “A probabilistic approach to evaluate strategies for selecting a parking space," Transportation Science, 32(1): 30-42.

D'Acierno, L., Gallo, M., and Montella, B. (2006), "Optimisation models for the urban parking pricing problem," Transport Policy, 13(1): 34-48.

Dragos, S.L. (2014), "Life and non-life insurance demand: the different effects of influence factors in emerging countries from Europe and Asia," Economic Research-Ekonomska Istrazivanja, 27(1):169180, 2014.

Elliot, J. and Wright, C. (1982), "The collapse of parking enforcement in large towns: Some causes and solutions," Traffic Engineering \& Control, 23(HS-033 448).

Fisman, R., and Miguel, E. (2007), "Corruption, norms, and legal enforcement: Evidence from diplomatic parking tickets," Journal of Political Economy, 115(6): 1020-1048.

Ganderton, P.T., Brookshire, D.S., McKee, M., Stewart, S., and Thurston, H. (2000), "Buying insurance for disaster-type risks: experimental evidence," Journal of Risk and Uncertainty, 20(3): 271289.

Hess, D.B. (2001), "Effect of free parking on commuter mode choice: Evidence from travel diary data," Transportation Research Record: Journal of the Transportation Research Board, 1753(1): 35-42.

Hung, H.-C. (2009), "The attitude towards of insurance purchase when respondents' preferences are uncertain: A fuzzy approach,” Journal of Risk Research, 12(2): 239-258.

Jeroslow, R. (1973), "There cannot be any algorithm for integer programming with quadratic constraints," Operations Research, 21(1): $221-224$.
Kannan, R., and Monma, C. (1978), "On the computational complexity of integer programming problems," in Optimization and Operations Research, vol. 157 of Lecture Notes in Economics and Mathematical Systems, edited by R. Henn, B. Korte, and W. Oettli, 161172, New York: Springer Berlin Heidelberg.

Kunreuther, H. (1996), "Mitigating disaster losses through insurance," Journal of Risk and Uncertainty, 12(2-3): 171-187.

Larson, R.C. (1970), "On the modeling of police patrol operations," Systems Science and Cybernetics, IEEE Transactions on, 6(4): 276-281.

Lewis, T., and Nickerson, D. (1989), "Self-insurance against natural disasters," Journal of Environmental Economics and Management, 16(3): 209-223.

Meier, V. (1999), "Why the young do not buy long-term care insurance," Journal of Risk and Uncertainty, 18(1): 83-98.

Miller, G.K., and Everett, C.T. (1982), "Raising commuter parking prices an empirical study," Transportation, 11(2): 105-129.

Mossin, J. (1968), “Aspects of rational insurance purchasing," Journal of Political Economy, 76(4, Part 1), 553-568.

Petiot, R. (2004), "Parking enforcement and travel demand management," Transport Policy, 11(4): 399-411.

Rojas, D. (2006), "Revenue management techniques applied to the parking industry," $\mathrm{PhD}$ thesis, University of South Florida.

Sherden, W.A. (1984), "An analysis of the determinants of the demand for automobile insurance," Journal of Risk and Insurance, 53(1): 49-62.

Shoup, D.C. (1995), "An opportunity to reduce minimum parking requirements," Journal of the American Planning Association, 61(1): $14-28$.

Shoup, D.C. (1997), "The high cost of free parking," Journal of Planning Education and Research, 17(1): 3-20.

Shoup, D.C. (1999), "The trouble with minimum parking requirements," Transportation Research Part A: Policy and Practice, 33 (7): 549-574.

Slovic, P., Fischhoff, B., Lichtenstein, S., Corrigan, B., and Combs, B. (1997), "Preference for insuring against probable small losses: Insurance implications," Journal of Risk and Insurance, 44(2): 237 258.

Verhoef, E., Nijkamp, P., and Rietveld, P. (1995), “The economics of regulatory parking policies: The (IM) possibilities of parking policies in traffic regulation," Transportation Research Part A: Policy and Practice, 29(2): 141-156.

Willson, R. W. (1995), "Suburban parking requirements: A tacit policy for automobile use and sprawl," Journal of the American Planning Association, 61(1): 29-42.

Willson, R. W., and Shoup, D. C. (1990), "Parking subsidies and travel choices: Assessing the evidence," Transportation, 17(2): 141-157. 
Copyright of INFOR is the property of University of Toronto Press and its content may not be copied or emailed to multiple sites or posted to a listserv without the copyright holder's express written permission. However, users may print, download, or email articles for individual use. 